\title{
COMUNICAÇÃo
}

\author{
[Communication]
}

\section{Spalangia drosophilae (Ashmead) (Hymenoptera: Pteromalidae) como inimigo natural de Archisepsis scabra (Loew) (Diptera: Sepsidae) em fezes bovinas}

\author{
[Spalangia drosophilae (Ashmead) (Hymenoptera: Pteromalidae) as natural enemy of \\ Archisepsis scabra (Loew) (Diptera: Sepsidae) in catlle dung]
}

\section{C.H. Marchiori}

Departamento de Biologia do Instituto Luterano de Ensino Superior de Itumbiara - ULBRA

Caixa Postal 23-T,

75.500-000 - Itumbiara, GO

Recebido para publicação em 23 de novembro de 2000

E-mail: Pesquisa@ns.itumbiara.com.br

A biologia da família Sepsidae é praticamente desconhecida para a região Neotropical. Sepsidae é normalmente encontrada próxima ou sobre fezes de animais ou em vários outros materiais em decomposição (Zuska, 1980; Silva, 1991; Amaral, 1996). As larvas desenvolvem-se em muitos materiais em decomposição, sendo freqüentes em fezes bovinas e carcaças de animais vertebrados (Steyskal, 1968).

Neste trabalho apresenta-se a primeira ocorrência do parasitóide Spalangia drosophilae em pupário de Archisepsis scabra em fezes bovinas coletadas no município de Cachoeira Dourada, GO. O experimento foi realizado na Fazenda Boa Vista, distante 20 quilômetros de Cachoeira Dourada (18 29'S' e 49²8'W'). A fazenda, com área aproximada de 114 hectares, possui 200 animais cruzados Holandês x Nelore destinados à produção de leite. As fezes foram expostas em pastagens são constituídas de Brachiaria brizantha (Hochst ex. A. Rich).

Fezes frescas marcadas imediatamente após sua emissão nas pastagens, com auxílio de estacas de madeira branca $(30 \mathrm{~cm}$ de altura e $5 \mathrm{~cm}$ de espessura) para determinação precisa do tempo desde a emissão, permaneceram no campo por oito dias. Posteriormente, foram coletadas e levadas para o laboratório do Instituto Luterano de Ensino Superior de Itumbiara, GO, para a extração das pupas pelo método da flutuação. Com as fezes eram retirados $5 \mathrm{~cm}$ do substrato abaixo. As pupas foram retiradas com o auxílio de uma peneira, contadas e individualizadas em cápsulas de gelatina (número 00) até a emergência das moscas e/ou dos parasitóides. Os parasitóides e as moscas emergidos foram identificados com auxílio de um microscópio esteroscópio e, posteriormente, conservados em álcool 70\%. Foram analisadas 84 placas de fezes para a obtenção de pupas. Este procedimento realizou-se quinzenalmente, de agosto a outubro de 2000.

A prevalência de parasitismo foi calculada segundo a fórmula de Margolis et al. (1982) e Bush et al. (1997).

Obtiveram-se 105 pupários de Archisepsis scabra (Loew) (Diptera: Sepsidae), das quais emergiram seis exemplares do parasitóide Spalangia drosophilae (Ashmead). A prevalência de parasitismo foi de 5,7\%. Outras espécies de Diptera coletadas foram: Braontaea debilis Williston, Brontaea quadristigma Thomson, Cyrtoneurina sp., Haematobia irritans (L.), Musca domestica L., (Muscidae), Ravinia belforti (Prado \& Fonseca), Sarcophagula sp. (Sarcophagidae), Palaeosepsis spp. (Sepsidae) e Sphaeroceridae sp. 
Os Sepsidae são as primeiras moscas a visitarem as fezes, mas provavelmente não se limitam somente a fezes frescas (Sanders \& Dobson, 1966). Essa afirmação é baseada no fato de que, aparentemente, algumas amostras produziram uma segunda onda de colonização. Mendes (1996) acredita que os Sepsidae são competidores da H.irritans, ovipondo e se desenvolvendo nas fezes no mesmo período. Larvas desse grupo contribuem para o processo de formação de túneis e aeração das fezes, facilitando a colonização e atuação de outros grupos de artrópodes envolvidos na desestruturação das placas fecais (Mendes, 1996). Hulley (1983) acredita que os Sepsidae possam ter importância na redução das moscas consideradas pragas caso haja competição ou outro tipo de interação.

Spalangia drosophilae é citada na literatura como parasitóide de pupas de dípteros pequenos das famílias Drosophilidae e Chloropidae (Boucek, 1963), mas também de H. irritans (Lindquist, 1936; Depner, 1968). Marchiori \& Linhares (1999) também coletaram esse parasitóide em pupários de Muscidae e Sarcophagidae e de outras espécies de Sepsidae (Palaeosepsis spp.)

Em laboratório, esse parasitóide foi observado em pupas de M. domestica (Boucek, 1963; Askew, 1971). Esse Pteromalidae é também hiperparasitóide, causando consideráveis danos em populações de Alysia ridibunda (Say) (Hymenoptera: Braconidae) (Lindquist, 1936).

O fato de se utilizar de muitos dípteros como mencionado acima, $S$. drosophilae favorece sua permanência no meio ambiente, aumentando o seu potencial como agente no controle biológico de espécies-pragas como provavelmente $H$. irritans.

Palavras-chave: Díptera, Hymenoptera, Spalangia drosophilae, parasitóide, fezes de bovinos, Goiás

\begin{abstract}
This paper reports, for the first time, the occurrence of the parasite Spalangia drosophilae (Hymenoptera: Pteromalidae) in pupae of Archisepsis scabra (Diptera: Sespsidae) found in cow manure in the

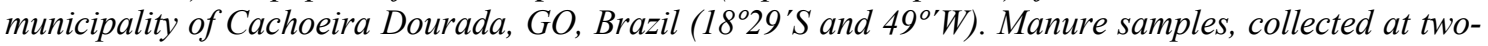
week intervals, were taken to the laboratory, and the pupae were extracted by water flotation. Each pupa was placed in capsules of colorless gelatin until the emergence of adult flies or their parasites. The parasitism prevalence was $5.7 \%$.
\end{abstract}

Keywords: Diptera, Hymenoptera, Spalangia drosophilae, parasites, cow manure, Brazil

\title{
REFERÊNCIAS BIBLIOGRÁFICAS
}

AMARAL, M.M.G. Dípteros simbovinos: colonização e sucessão em placas isoladas de fezes bovinas. Campinas: UNICAMP, 1996. 66p. (Dissertação, Mestrado).

ASKEW, R.R. Parasitic insects. London: Heineman Educational Books, 1971. 316 p.

BOUCEK, Z. A Taxonomic study in Spalangia Latr. (Hymenoptera: Chalcidoidea). Acta. Ent. Mus. Nat. Pragae, v.35, p.429-512, 1963.

BUSH, A.O, LAFFERTY, K.D., LOTZ, J.M. et al. Parasitology meets ecology on its own terms: Margolis et al. Revisited. J. Parasitol., v.83, p. 575-583, 1997.

DEPNER, K.R. Hymenopterous parasites of the horn fly, Haematobia irritans (Diptera: Muscidae), in Alberta. Can. Ent., n.100, p.1057-1060, 1968.

HULLEY, P.E. A survey of flies breeding in poultry manure, and their potencial enemies. J. Entomol. Soc. Sth. Afr., v.46, p.37-47, 1983.

LINDQUIST, A.W. Parasites of horn fly and other flies breeding in dung. J.Econ. Entomol., v.29, p.1154-1158, 1936. 


\section{Marchiori, C.H.}

MARCHIORI, C.H., LINHARES, A.X. Dípteros muscóides associados a fezes frescas de gado bovino e seus parasitóides. Braz. J. Ecol., v.3, p.26-31, 1999.

MARGOLIS, L., ESCH, G.W, HOLMES, J.C. et al. The use of ecological terms in parasitology (report of an ad hoc committee of the American Society Parasitologists). J. Parasitol., v.68, p-131-133, 1982.

MENDES, J. Sazonalidade da artropodofuana associada a fezes bovinas em pastagens e alguns aspectos da biologia dos estágios imaturos de Haematobia irritans (Linnaeus, 1758) na região de São Carlos, SP. Campinas: UNICAMP, 1996. 126p. (Tese, Doutorado).

SANDERS, D.P., DOBSON, R.C. The insect complex associated with bovine manure in Indiana. Ann. Entomol. Soc. Am., v.59, p.955-959, 1966.

SILVA, V.R. Levantamento preliminares de Sepsidae (Diptera: Schizophora) em Roraima, projeto Maracá com descrição de uma espécie nova. Acta Amazonica, v.21, p.369-374, 1991.

STEYSKAL, G.C. Family Sepsidae. In: Cataloque of the Diptera of the Americas South of the United States. São Paulo: Secretaria Agricultura 1968, 6p.

ZUSKA, J. Family Sepsidae. In: Cataloque of the Diptera of the Afrotropical Region. London: British Museum, 1980. p.591-596. 